\title{
Practical Issues in Diagnostic Neuropathology: It Is Not Even the End of the Beginning!
}

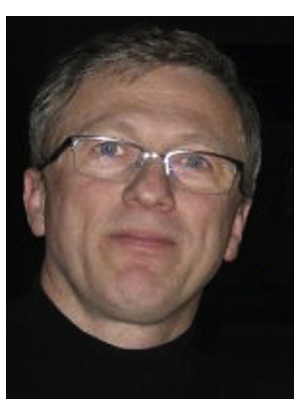

Tarik Tihan, MD, PhD

Editor
Classification schemes are often dynamic, living paradigms that periodically undergo expansion or shrinkage, fragmentation or coherence, and yet, inescapably become more comprehensive and multidimensional. What started as mere interpretations of the light microscopic images in the last century are now facing significant modifications through the use of a spectrum of advanced analytical tools. Classification of the diseases of the central nervous system (CNS), and in particular, tumors and paraneoplastic diseases, is no exception. The last two decades have seen tremendous progress in advanced technology, such as MRI and molecular biology, and it is no longer possible to ignore the wealth of information provided by these technologies in the understanding, diagnosis, and treatment of CNS diseases.

Until a decade ago, it was mostly sufficient to provide the type and the grade of a CNS neoplasm based on the routine H\&E stains alone, and this bit of information was helpful in an overwhelming majority of tumors. In fact, the neuro-oncologists did not have specific treatment for most of the tumor types or variants in the WHO classification scheme. Some entities, variants, or families of tumors were being managed in the same fashion, so a lengthy debate about whether a neoplasm should be considered oligoastrocytoma or astrocytoma was only relevant at the microscope, but not at the bedside. Our occasionally subjective interpretations and discrepancies in neuropathology were of little consequence, and the pathologic interpretation was useful as long as it gave the treating physician a general idea on the nature of the disease.

Well, that era is over! It has been brought to an end by the expanding number of advanced treatment options, diagnostic methodologies, and the attempts to "personalize" patient management. It is time to recognize the complexities of biology that give us a spectrum of genotypic and phenotypic attributes of the same pathologic process and to give equal importance to both. It is still critical to recognize the phenotypic attributes of a disease as reflected under the microscope. Yet, mere phenotypic characterization is increasingly becoming incomplete as we understand the other side of the coin. It is time for the pathologists to add a few more weapons to their diagnostic arsenal other than the faithful light microscope.

The articles in this issue of Surgical Pathology Clinics are a testament to the fact that characterization of tumors above and beyond the information provided on routine histological stains is no longer a feat of "fancy" academic centers alone. Today, the practicing pathologists have to incorporate the information that was mostly limited to reports from advanced academic centers only a decade ago. It is with this realization that the authors in this issue have provided their expertise 
and experience to practicing pathologists at all levels.

The first article by Glastonbury et al is a gentle warning to practicing pathologists that neuropathologic interpretation can be significantly aided by a good understanding of neuroradiology, which can be achieved through collaboration with a neuroradiologist. The importance of intraoperative consultation and how it can be practiced with the utmost benefit to the patients are highlighted in the article by Lee. The articles by Pekmezci et al and by Reis et al underscore the need to include a set of critical molecular markers in the diagnosis of both diffuse and solid glial neoplasms. The markers detailed in these articles are no longer "luxury items" and will need to be available for the upcoming standard of care in surgical neuropathology. Substantive changes in the understanding of diffuse glial neoplasm are imminent, and these will provide a significant modification of the existing WHO 2007 scheme. Phillips and colleagues briefly summarize the impressive amount of progress on embryonal tumors that took place in the last few years. It is important to recognize the markers that will be asked of the practicing pathologists when reporting CNS embryonal tumors. Finally, Dr Gultekin, a world expert in the diagnosis and evaluation of paraneoplastic diseases in the CNS, provides a comprehensive and enlightening review of these enigmatic entities. His tables and figures are very helpful in understanding the new developments, and the article provides a beautiful template for aiding the workup of these challenging entities.

I am deeply indebted to all the authors for the time and the effort they have put into this issue. I also wish to acknowledge the hundreds of excellent scientists who have reported their results in numerous publications, recent or remote. Without their collective effort of adding one building block of information at a time, we would have little, if any, understanding of the mechanisms of CNS diseases, their pathogenesis, treatment, or prognosis. I feel proud to be able to work with all the authors to bring a synopsis of these advances to our readers. This feeling was best formulated a few centuries ago by Sir Isaac Newton: "If I have seen further, it is by standing on the shoulders of giants." We can look further ahead together to see what is beyond the next hill.

Tarik Tihan, MD, PhD Neuropathology Division Department of Pathology UCSF School of Medicine UCSF Medical Center Room M551

505 Parnassus Avenue San Francisco CA 94143-0102, USA

E-mail address: Tarik.Tihan@ucsf.edu 